\title{
Comparison of the Performance of Skin Prick, ImmunoCAP, and ISAC Tests in the Diagnosis of Patients with Allergy
}

\author{
Rebecca L.M. Griffiths Tariq El-Shanawany Stephen R.A. Jolles Clive Selwood \\ Adrian G. Heaps Emily M. Carne Paul E. Williams \\ Department of Immunology, University Hospital of Wales, Cardiff, UK
}

\section{Keywords}

Allergy diagnosis - Sensitivity - Specificity - Skin prick tests . Allergen-specific IgE (ImmunoCAP) tests · ISAC allergen array $\cdot$ Component-resolved diagnostics

\begin{abstract}
Background: Allergy is diagnosed from typical symptoms, and tests are performed to incriminate the suspected precipitant. Skin prick tests (SPTs) are commonly performed, inexpensive, and give immediate results. Laboratory tests (ImmunoCAP) for serum allergen-specific IgE antibodies are usually performed more selectively. The immuno-solid phase allergen chip (ISAC) enables testing for specific IgE against multiple allergen components in a multiplex assay. Methods: We retrospectively analysed clinic letters, case notes, and laboratory results of 118 patients attending the National Adult Allergy Service at the University Hospital of Wales who presented diagnostic difficulty, to evaluate which testing strategy (SPT, ImmunoCAP, or ISAC) was the most appropriate to use to confirm the diagnosis in these complex patients, evaluated in a "real-life" clinical service setting. Results: In patients with nut allergy, the detection rates of SPTs (56\%) and ISAC (65\%) were lower than those of ImmunoCAP (71\%). ISAC had a higher detection rate (88\%) than Immuno-
\end{abstract}

\section{KARGER}

() 2017 S. Karger AG, Basel

E-Mail karger@karger.com

www.karger.com/iaa
CAP $(69 \%)$ or SPT (33\%) in the diagnosis of oral allergy syndrome. ImmunoCAP test results identified all 9 patients with anaphylaxis due to wheat allergy (100\%), whereas ISAC was positive in only 6 of these 9 (67\%). Conclusions: In this difficult diagnostic group, the ImmunoCAP test should be the preferred single test for possible allergy to nuts, wheat, other specific foods, and anaphylaxis of any cause. In these conditions, SPT and ISAC tests give comparable results. The most useful single test for oral allergy syndrome is ISAC, and SPT should be the preferred test for latex allergy.

(c) 2017 S. Karger AG, Basel

\section{Introduction}

There is a worldwide increase in the prevalence of allergy, presumed to be due to environmental factors acting on the more genetically susceptible individuals in the population. The diagnosis of clinical allergy presents an increasing burden to medical systems worldwide and there is thus a need to review critically how well currently available diagnostic tests perform, how they are used and how their use might be optimised. In some health systems there is still a large unmet need in allergy diagnosis [1-4]. 
Allergy is diagnosed on the basis of the history of the symptoms as given by the patient. Tests are performed to seek to confirm the clinical suspicion of allergy to the likely, suspected precipitating agent. Tests are also sometimes performed to attempt to distinguish between the most likely agents where 2 or 3 different possible precipitants are suspected of causing symptoms. This is important, for example, in patients who have respiratory symptoms all year round and keep pets, in order to try and see to what extent allergy to pets and/or house dust mite may be implicated in causing their symptoms. Patients who have had symptoms suggestive of allergy after food may not always be able to identify the precipitating food with certainty, especially if mixtures of different foods have been eaten prior to the occurrence of symptoms or if there is a significant delay between food ingestion and symptoms, as may occur in food-dependent exercise-induced anaphylaxis.

The prevalence of nut allergy was reported as being at least $1 \%$ in 2010 , and this rate is doubling approximately every 10 years [5]. Tests for IgE antibodies that bind specifically to recombinant or native purified allergen components (rather than whole allergen extracts) now enable individual risk stratification. This has been shown to provide useful information about the probability of reactions occurring following challenge with those nuts, and the likely severity of reactions if they should occur [6]. An increasing number of blood tests are emerging that can identify such components, termed component-resolved diagnostics.

The oral allergy syndrome (also known as the pollen food syndrome) seems to be increasing in prevalence. It is currently reported in UK primary care practices as affecting approximately $2 \%$ of the population [7]. Although this can often easily be diagnosed from the history, there is significant demand for confirmatory tests from patients who have typical oral symptoms after eating raw fruit or vegetables and sometimes raw nuts. The overlap in symptoms between patients who have the oral allergy syndrome and patients who have nut allergy is often an area that requires a more precise definition using currently available tests in order to stratify risk and inform patient management. Tests for an increasing number of recombinant proteins of relevance are becoming available for use both in the ImmunoCAP format and in the ISAC format, and these are particularly helpful in clarifying whether patients react only to PR-10 and/or profilin proteins (with an associated lower risk of severe clinical reactions) or to lipid transfer or nut storage proteins (which have a higher risk of severe reactions).

216

Int Arch Allergy Immunol 2017;172:215-223 DOI: $10.1159 / 000464326$
Skin prick tests (SPTs) are commonly performed in outpatient clinics worldwide as the required materials are relatively cheap and give immediate results. Tests for allergen-specific IgE antibodies in the blood, such as ImmunoCAP tests, are usually performed more selectively and only when clinically relevant. Other blood testing systems that are available include the HYCOR HYTEC 288 Plus system that uses activated cellulose solid-phase technology, and the IMMULITE ${ }^{\circledR} 2000$ and IMMULITE $^{\circledR} 2000$ XPi Immunoassay allergy testing systems that use Siemens 3gAllergy Liquid Allergens Testing technology. All of these detect IgE antibodies of both low and high affinity as the allergens are present in excess of serum-specific antibody in the assay. Recently, microarray assay formats have been developed where specific IgE antibodies against multiple allergens can be tested in the same small volume $(30 \mu \mathrm{L})$ of serum. The immuno-solid phase allergen chip (ISAC) test (Thermo Fisher ImmunoCAP ISAC) is a microarray assay system designed to assay specific IgE antibodies against multiple recombinant allergen proteins ( 112 in its current iteration) in a multiplex assay that uses a small volume of serum. Serum is in excess of the allergen and thus IgE antibodies of high affinity are the ones which predominantly bind, in contrast to the ImmunoCAP test which detects IgE antibodies of both high and low affinity [8]. For reasons of cost, in most clinical services in the UK at present, ISAC tests are only conducted in selected patients in whom diagnostic uncertainty remains even after SPTs and ImmunoCAP tests have been performed. Outside the UK, some clinicians report that performing ISAC tests as a matter of routine is promising [9].

In view of the evolution of clinical diagnostic practice in allergy, we performed a retrospective review of the clinical histories and the results of all relevant allergy tests of patients who presented diagnostic difficulty (defined in this report as those in whom diagnostic uncertainty remained after being seen by a consultant and after SPT and ImmunoCAP tests have been performed) in order to inform the choice of the clinically most useful and costeffective testing strategies to use in different clinical presentations.

\section{Subjects and Methods}

Patients

In total, 1,434 female and 634 male patients attended the secondary and tertiary National Allergy Service for Wales at the University Hospital of Wales as new referrals between April 4, 2011, and March 31, 2014 (female to male ratio of 2.26). In this service,

Griffiths/El-Shanawany/Jolles/Selwood/ Heaps/Carne/Williams 
as for most current routine UK NHS clinical practice, SPTs are used as the first-choice confirmatory investigation. Patients attending the service routinely have a defined panel of SPTs performed prior to their clinical consultation with an NHS consultant as this enables more patients to be seen in each clinic by streamlining their patient pathway. The standard panel of SPT reagents used on all patients was histamine, saline control, house dust mite, cat, dog, mixed grass pollens, mixed tree pollens, egg, milk, peanut, hazelnut, almond and walnut. Other reagents used in selected patients were: Aspergillus fumigatus, latex, cod, shrimp, tuna, birch pollen, kiwi fruit, wasp venom, bee venom and horse dander. The second line investigation utilised by this service is the ImmunoCAP test, used to measure allergen-specific IgE. This test is performed according to clinical need and informed by the history. ISAC microarray tests are reserved in this service for patients in whom there remains diagnostic difficulty despite the results of relevant SPT and ImmunoCAP tests, where response to a larger panel of allergens may give helpful diagnostic information in complex clinical presentations of symptoms following exposure to multiple allergens, or where it is less expensive to perform an ISAC test than multiple single allergen ImmunoCAP tests. Of the total number attending the service, 118 patients ( 90 female and 28 male; female to male ratio of 3.21) fell into 1 of the above categories and underwent ISAC testing, thus forming the study population. Of these, 117 were adults and 1 was aged under 16 years. All patients in the study population had been referred to the National Allergy Service either by their general practitioners or by hospital practitioners. Ethical permission and patient consent for the retrospective review of normal clinical practice is not required and was not sought.

\section{Skin Prick Tests}

All SPT reagents were purchased from Allergy Therapeutics (Worthing, UK) apart from almond, kiwi, Aspergillus fumigatus and tuna from Stallergenes SA France, and peanut, walnut, wasp, bee, latex, shrimp and horse dander from ALK-Abelló UK. ImmunoCAP tests were done on the Thermofisher Phadia 250 platform. ISAC tests were performed using the Thermo Fisher ISAC system [8].

\section{ImmunoCAP}

Routine serum measurements of specific IgE were performed using a fluoroenzyme immunoassay auto-analyser, the ImmunoCAP 250 platform (ThermoFisher), according to the manufacturer's guidelines. Established cut-off values were used with respect to ImmunoCAP results: those with a result of $0-0.34 \mathrm{KUA} / \mathrm{L}$ were considered not to be sensitised to the allergen in question and those with a result of $>0.35 \mathrm{KUA} / \mathrm{L}$ were considered to be sensitised.

\section{ISAC}

The allergen microarray assay (ImmunoCAP ISAC; ThermoFisher) was used to analyse the specific IgE repertoire of each patient's serum. ISAC reactions were performed according to the manufacturer's instructions [8]. Processed ISAC slides were scanned using a GenePix4000B microarray scanner (Molecular Devices, Sunnyvale, CA, USA) and image acquisition was performed using GenePix Pro (Molecular Devices). Image analysis was performed using microarray image analyser software (MIA, ThermoFisher).

Allergy Microarray Tests in Patients

Presenting Diagnostic Difficulty
Table 1. Sensitivity and specificity of different tests

\begin{tabular}{|c|c|c|c|c|c|}
\hline & \multicolumn{4}{|l|}{ Disease } & \multirow[t]{2}{*}{ Total } \\
\hline & present & $n$ & absent & $n$ & \\
\hline \multicolumn{6}{|l|}{ Test } \\
\hline Positive & true positive & $\mathrm{a}$ & false positive & c & $a+c$ \\
\hline Negative & false negative & $\mathrm{b}$ & true negative & $\mathrm{d}$ & $\mathrm{b}+\mathrm{d}$ \\
\hline Total & & $a+b$ & & $c+d$ & \\
\hline
\end{tabular}

"Disease" refers to the disorder in question. "Test" refers to the diagnostic test (SPT, ImmunoCAP, or ISAC). Sensitivity and specificity were calculated as shown in the formulae. Sensitivity $=$ $a /(a+b)$. Specificity $=d /(c+d)$.

\section{Diagnostic Categories}

The performance of the confirmatory diagnostic tests used was analysed in defined patient clinical diagnostic categories. The categories were nut allergy, oral allergy syndrome, IgE-mediated symptoms after other food, anaphylaxis, latex allergy, and rhinitis and/or conjunctivitis, defined on the basis of the referral letters and medical notes available from primary and secondary care. Nut allergy was diagnosed clinically on the basis of typical symptoms after the ingestion of nuts. The oral allergy syndrome was diagnosed clinically on the basis of typical oral symptoms after eating raw fruit or vegetables and sometimes raw nuts.

\section{Calculation of Test Performance}

We calculated the detection rate of a test as the percentage of test results that confirmed the patient's clinical diagnosis as determined by the patient's history. We did not perform any statistical tests of the difference between the detection rate of 1 diagnostic test and another in view of the absence of the gold standard result of challenge testing. The sensitivities and specificities of SPT, ImmunoCAP and ISAC were calculated for patients in each diagnostic category, as shown in Table 1 . We did not calculate these 2 parameters for combinations of tests as, for some patients, diagnostic tests gave conflicting results. In these instances, it would be difficult to categorise outcomes adjudged to give false-positive or falsenegative results.

\section{Results}

Figure 1 details the number of patients of each sex in each diagnostic group. Online supplementary Table 1 (see www.karger.com/doi/10.1159/000464326) lists relevant anonymised details of all patients, including their sex, age when seen, their primary (and if applicable) secondary and tertiary diagnoses, and their SPT, ImmunoCAP and ISAC results.

Figure 2 displays the detection rates of SPTs, ImmunoCAP tests and ISAC tests as well as various combinations

Int Arch Allergy Immunol 2017;172:215-223 
of these tests for patients belonging to different diagnostic groups and subgroups. We defined the detection rate as the percentage of test results that confirm the clinical diagnosis made from the patient's history. When calculating the detection rates for combinations of tests, we included only those patients who had had the relevant combinations of tests performed.
Figure 3 presents the sensitivities and specificities of each of the 3 individual test methodologies for patients belonging to each relevant diagnostic group. Absent histogram bars indicate instances where the numerator ( \pm the denominator) for the relevant calculation was " 0 ," which therefore prevented calculation of the aforementioned parameters.

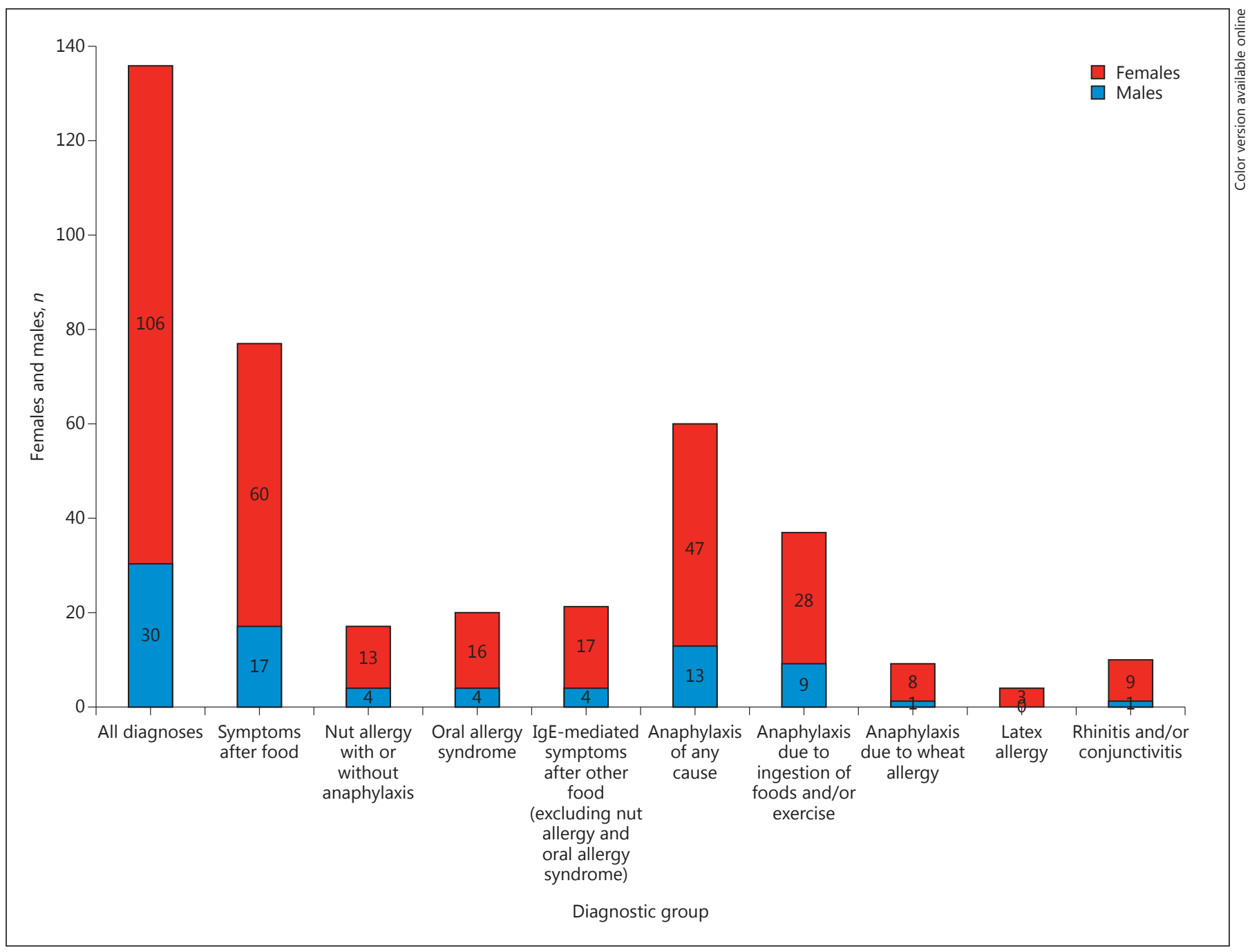

Fig. 1. The number of females and males in each diagnostic group. Patients who had either anaphylaxis (major disturbance of airway, breathing, or circulation) or throat tightness were included in the same clinical diagnostic group.

Fig. 2. Comparison of the detection rates of SPTs, CAP, and ISAC tests, and of combinations of these tests. The detection rate is the percentage of test results that confirm the clinical diagnosis made from the patient's history. These rates are shown for patients grouped into the major different diagnostic groups. Patients who had either anaphylaxis (major disturbance of airway, breathing or circulation) or throat tightness are included in the same clinical diagnostic group. The percentages refer to the number testing positive using the test or combination of tests.

(For figure see next page.) 


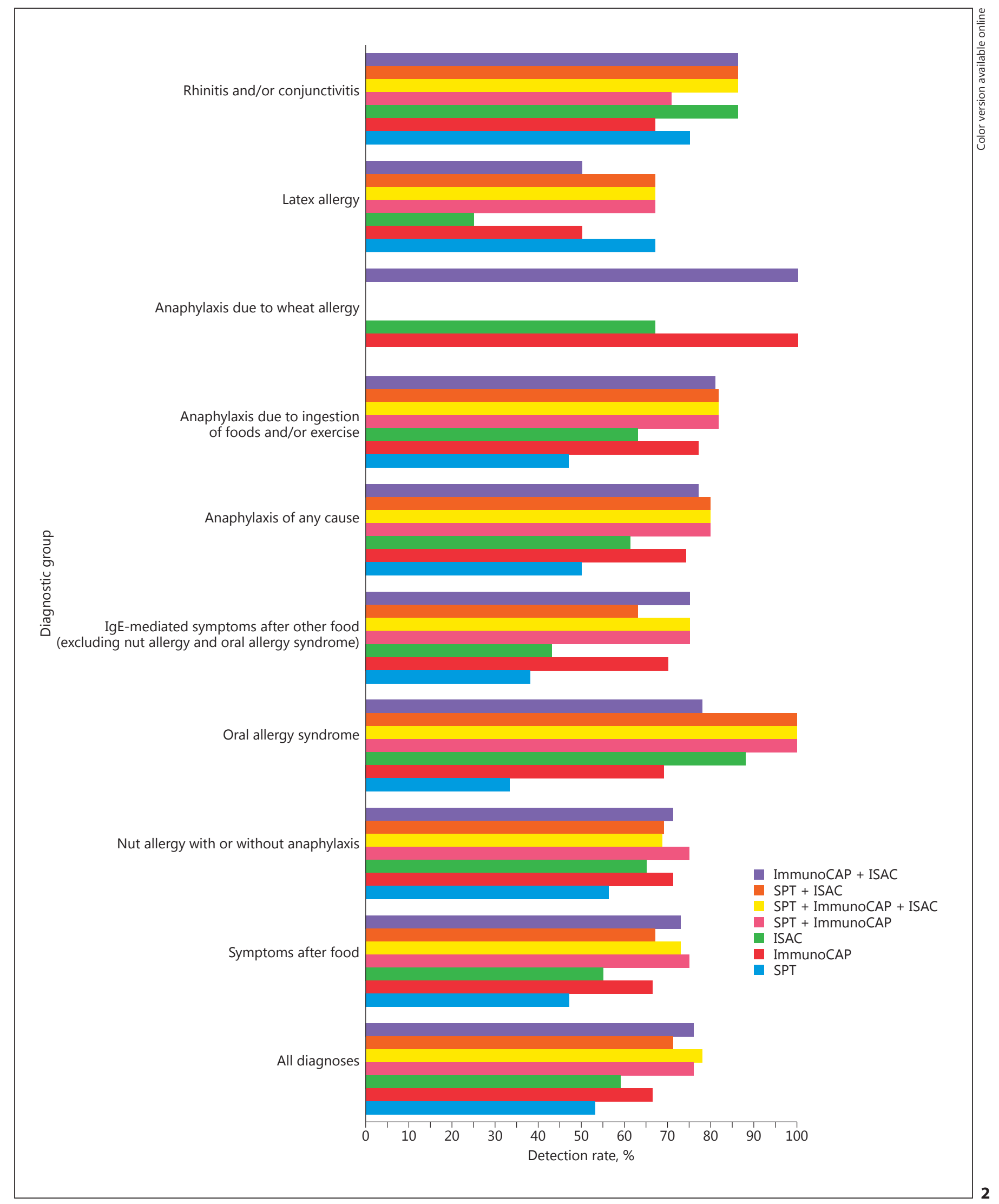

Allergy Microarray Tests in Patients

Int Arch Allergy Immunol 2017;172:215-223 


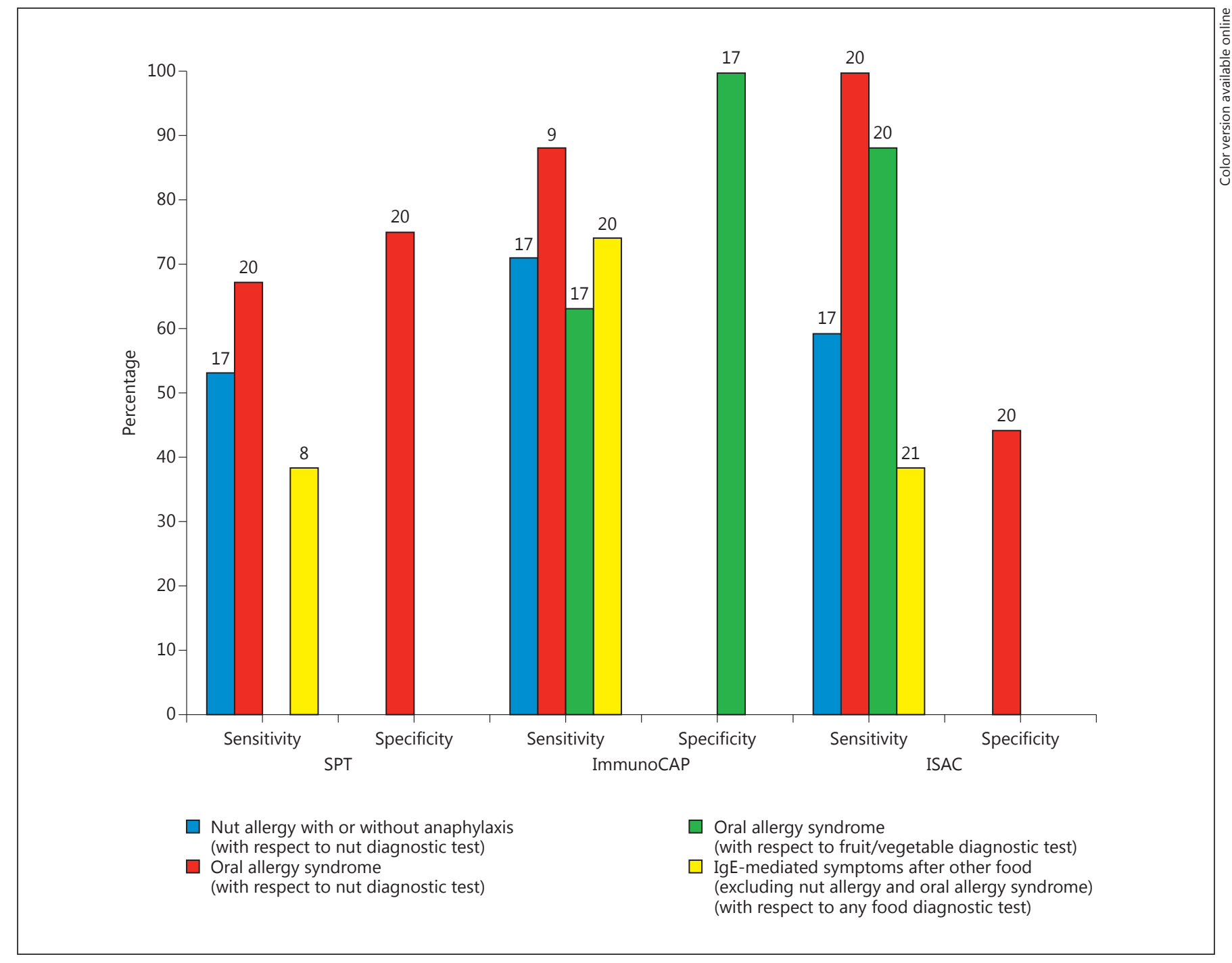

Fig. 3. The sensitivity and specificity of SPTs, ImmunoCAP, and ISAC tests. These rates are shown for patients grouped into the major different diagnostic groups. The numbers above each histogram bar show the corresponding number of patients in the diagnostic group who had that particular test performed. Absent histogram bars indicate instances where the numerator $( \pm$ the de- nominator) for the relevant calculation was " 0 ," which therefore prevented calculation of the aforementioned parameters. No results are given for SPT for the oral allergy syndrome patient group with respect to testing for fruit and vegetables as these patients did not have a SPT performed with fruit (other than kiwi fruit, if relevant), according to the normal practice of the service.
Laboratory confirmatory evidence was present in 13 of the 17 patients diagnosed as having nut allergy. In the remaining 4 there was a highly convincing history of nut allergy without such laboratory confirmatory evidence. Laboratory confirmatory evidence was present in 17 of the 20 patients diagnosed as having oral allergy syndrome. In the remaining 3 there was a highly convincing history of oral allergy syndrome without such laboratory confirmatory evidence.
Sensitisation to hazelnut was confirmed by ImmunoCAP and/or ISAC tests in 38 patients but only 25 of these had SPT reactivity to hazelnut. The remaining 13 patients had positive ImmunoCAP and/or ISAC test results with negative SPT findings. No patient had a positive SPT result for hazelnut and negative ImmunoCAP and ISAC test results.
Griffiths/El-Shanawany/Jolles/Selwood/ Heaps/Carne/Williams 


\section{Discussion}

The female to male ratio of 2.26 is typical for this allergy service. The 118 patients selected for ISAC testing were those with more complex clinical presentations and who presented diagnostic difficulty. This study population comprised 90 females and 28 males, giving a female to male ratio of 3.21. This relative preponderance of females at the more complex end of the clinical spectrum may be consistent with the higher proportion of females attending the service in general. It is unclear to what extent this may represent referral bias, ascertainment bias, or a true biological sex difference in prevalence.

Of the 118 patients studied, many had medical conditions that could not be diagnosed by some of the test methods used. For patients with salicylate-induced or drug-induced urticaria or angioedema there are no confirmatory tests. Such patients were thus excluded from the calculation of detection rates, leading to the values for the number of patients in each different patient diagnostic category $(n)$ shown in Figure 2 all being well below 118. The number of diagnoses (136) exceeds the number of patients described in this report (118) as many patients had more than 1 allergy-related diagnosis; these diagnoses were considered separately within the analysis.

The major diagnostic categories in Figure 2 are split into subgroups where relevant. The number of patients in each diagnostic category ranges from 3 patients tested by SPTs in the "oral allergy syndrome" subgroup and the "latex allergy" major diagnostic category to 70 patients tested by the ISAC in the "all diagnoses" major category. The "all diagnoses" category comprises only those patients in whom SPTs or blood tests might be able to confirm the clinical diagnosis, and excludes those patients who may have had salicylate or drug-induced symptoms which these tests do not detect.

For the "all diagnoses" category the detection rates for SPTs (53\%) and ISAC (59\%) were similar, with a higher detection rate for ImmunoCAP testing (66\%). This applies to most of the clinical diagnostic categories throughout Figure 2 and may reflect the general clinical reliance made upon the ImmunoCAP test. The ISAC array has a higher detection rate (88\%) than the ImmunoCAP test $(69 \%)$ or SPT $(33 \%)$ in the diagnosis of oral allergy syndrome. The low detection rate of SPTs in oral allergy syndrome is due to the lack of availability of component-resolved diagnostics, and in particular PR-10, which limits the diagnostic yield. ISAC testing in this group may have had a higher rate $(88 \%)$ of detection than ImmunoCAP

Allergy Microarray Tests in Patients Presenting Diagnostic Difficulty tests $(69 \%)$ due to the more selective use of fewer allergens by ImmunoCAP testing compared with the larger number of relevant allergens included in each ISAC test. In patients with nut allergy with or without anaphylaxis, the detection rates of SPTs (56\%) and ISAC (65\%) are lower than those using ImmunoCAP tests (71\%). Seventeen patients were diagnosed as having nut allergy, and the ISAC test confirmed nut sensitisation in 11 of these. Four of the 6 patients that tested negative by ISAC also tested negative on both SPT and ImmunoCAP tests. In these 4 the clinical diagnosis was made on the basis of a definite clinical history, without confirmatory challenge testing. The remaining 2 ISAC-negative patients both tested positive for nuts on ImmunoCAP testing, and 1 was negative on SPT. To some extent, it is possible that the higher rate of clinical confirmation found by ImmunoCAP tests may reflect the clinical relevance of antibodies of lower affinity which the ImmunoCAP test detects (as allergen is present in excess in this testing system) but which the ISAC test may not detect.

SPTs and blood tests are used in order to confirm a clinical diagnosis of allergy made on the basis of the history of symptoms and events. In those patients with a clinical diagnosis of food allergy, it would have been most helpful to have performed oral food challenge tests to prove their diagnosis. Food challenge tests were not performed in these patients due to the lack of resources available to perform food challenge testing in our clinical service (which has no dietetic support). In view of this service limitation, during clinical consultations with patients presenting with food-related symptoms, the history of such symptoms was probed very thoroughly by the consulting specialist physician in order to confirm that all such patients gave a clear-cut history of symptoms that indicated food allergy.

When combinations of tests are considered, the detection rates of different combinations in the different patient diagnostic groups are broadly very similar. In patients who have nut allergy with or without anaphylaxis caused by nut allergy, a detection rate of around $70 \%$ is found by these test combinations, which is very similar to the detection rate of $71 \%$ found by the ImmunoCAP test alone, and higher than the $56 \%$ found by SPT alone. In patients with the oral allergy syndrome, the highest detection rates are achieved by the ISAC array, which includes a large number of the causative PR10 Bet $\mathrm{v} 1$ homologue and profilin Bet $\mathrm{v} 2$ homologue allergen probes. The group of patients with IgE-mediated symptoms after specific foods other than nuts or fruits causing the oral allergy syndrome had detection rates of $63-75 \%$ using

Int Arch Allergy Immunol 2017;172:215-223 221 
combinations of tests, similar to the $70 \%$ detection rate of the ImmunoCAP test alone. For patients presenting with anaphylaxis, combinations of tests gave detection rates in the order of $80 \%$. This is similar to that achieved by the ImmunoCAP test alone (74\%), and superior to SPT (50\%) as used in this service. ImmunoCAP test results identified all 9 patients who were diagnosed with anaphylaxis due to wheat allergy (100\%), whereas the ISAC test was positive in only 6 of these 9 patients (67\%). The corresponding result for SPTs cannot be interpreted as the clinic does not use the wheat SPT reagent as this has previously been found by us to give an unacceptably high rate of false-positive reactions in people who tolerate wheat normally.

Four patients had a clinical diagnosis of latex allergy. Three had an SPT against latex performed, of whom 2 tested positive, while 4 had ImmunoCAP tests, of whom 2 tested positive, and 4 had ISAC tests, of whom 1 tested positive. This indicates that patients with a suspected diagnosis of latex allergy should have SPTs and ImmunoCAP tests performed as part of their assessment, but there were too few patients to enable firm conclusions to be drawn about optimal testing for latex allergy. All tests gave similar detection rates for rhinoconjunctivitis (SPTs 75\%, ImmunoCAP 67\%, and ISAC 86\%).

In patients with a diagnosis of nut allergy (with or without anaphylaxis; Fig. 3), tests for sensitisation to nuts performed similarly, but with a greater sensitivity (71\%) for ImmunoCAP tests than SPT (53\%) or ISAC (65\%). For patients with a diagnosis of oral allergy syndrome, tests for sensitisation to nuts were most sensitive using ISAC (100\%) or ImmunoCAP (88\%) rather than SPT (67\%). This could be due to the superior performance of recombinant allergens compared with SPT in the context of oral allergy syndrome. For patients with a diagnosis of oral allergy syndrome, ImmunoCAP and ISAC tests for sensitisation to fruit and vegetables perform similarly.

In the 21 patients with a history indicating IgE-mediated allergy to specific foods other than nuts and excluding the oral allergy syndrome, ImmunoCAP tests appeared to be more sensitive (73\%) than ISAC (38\%) or SPT (37\%) in confirming the clinical diagnosis. This is in part due to SPT reagents for many of these specific foods (e.g., chicken, turkey, etc.) not being used, partly due to ImmunoCAP testing being more feasible in this situation, and partly due to many of these foods not being represented in the ISAC test.

When no cause for anaphylaxis is apparent from the history, SPT or targeted ImmunoCAP tests (i.e., idiopath- ic anaphylaxis), the ISAC test can contribute to making a causal diagnosis in $20 \%$ of patients. It may offer additional information where a careful allergy history and followon SPT and ImmunoCAP tests have not revealed the cause of the anaphylaxis, and so is an important test to add in this situation [10].

Other studies of the performance of the ImmunoCAP and ISAC tests have been of patients with defined clinical syndromes so as to compare the diagnostic performance of specific allergen tests, rather than being an evaluation of the use of these methodologies in a heterogeneous group of patients that present diagnostic difficulty to diagnostic services. For example, ImmunoCAP and ISAC tests have a similar sensitivity and specificity in patients diagnosed with grass and cypress pollen allergy on the basis of clinical history and SPTs [11]. SPT and ImmunoCAP tests are generally comparable in predicting or defining symptomatic food hypersensitivity [12]. ImmunoCAP and microarray tests have equivalent performance in the diagnosis of allergy to grass and birch pollens and cat [13]. The concordance between SPT and in vitro specific IgE antibody assays is between 85 and $95 \%$ depending on the allergen tested and the method used to detect specific IgE [14].

Sensitisation to hazelnut was detected by SPT in only 25 of the 38 patients found positive by ImmunoCAP and/ or ISAC testing, without any patients having positive hazelnut SPT results with negative ImmunoCAP and ISAC test results. There is known to be continuing variability in the composition of commercially available allergen extracts for SPT use between different manufacturers. For example, there is up to a 10 -fold variability in the total protein concentration and in the content of the major birch pollen allergen Bet $v 1$ [15]. This argues in favour of using in vitro testing or alternatively the use of recombinant allergen-based preparations, particularly in view of the reproducibility of such assays [16].

\section{Conclusions}

Unlike previous studies, we have evaluated the use of 3 tests in a "real-life" clinical service setting with heterogeneous categories of patient referrals that present diagnostic difficulty, as would be encountered in a secondary and tertiary allergy service. For the population assessed, we found the ImmunoCAP test to be the most suitable single test for the confirmation of allergy to nuts, wheat and other specific foods, and anaphylaxis of any cause. In these conditions SPT and ISAC tests give comparable re- 
sults. The most useful single test for oral allergy syndrome is the ISAC test, and SPT is the preferred test for latex allergy. However, our findings should be interpreted with care; the performance of diagnostic tests may vary from one geographic population to another, and within any population will vary among different groups of patients selected on clinical criteria.

\section{Acknowledgements}

The authors wish to thank Bethan Lee, Lisa Thomas, Nichaela Meredith, and Dawn Davey for maintaining the patient clinical database, and Clare Kingdon and Charis Joyce for their help in the clinics. Stephen Jolles is supported by a NISCHR fellowship.

\section{Disclosure Statement}

P.E.W. and T.E. received remuneration for attendance at a ThermoFisher Scientific advisory board. T.E. received support for conference/educational event attendance from ALK Abello, Stallergenes and Allergy Therapeutics. S.R.A.J. and A.G.H. received project and conference support from ThermoFisher Scientific.

\section{Author Contributions}

P.E.W., S.R.A.J., and T.E. designed the study, R.L.M.G. analysed all the data and wrote the first manuscript drafts, A.G.H. performed the ISAC tests, C.S. performed all other laboratory assays, and E.M.C. performed SPTs and helped look after the patients. All authors reviewed the manuscript.

\section{References}

1 Royal College of Physicians: Allergy: the unmet need - a blueprint for better patient care. Report of a working party. London, RCP, 2003.

2 House of Commons Health Committee: The provision of allergy services. Sixth report of session 2003-04. London, TSO, 2004.

3 House of Lords Science and Technology Committee: Allergy: sixth report of session 2006-07. London, TSO, 2007.

4 Royal College of Physicians; Royal College of Pathologists: Allergy: still not meeting the unmet need. Report of a joint colleges working party. London, RCP, 2010.

5 Sicherer SH, Muñoz-Furlong A, Godbold JH, Sampson HA: US prevalence of self-reported peanut, tree nut, and sesame allergy: 11-year follow-up. J Allergy Clin Immunol 2010;125: 1322-1326.

6 Nicolaou N, Poorafshar M, Murray C, Simpson A, Winell H, Kerry G, Härlin A, Woodcock A, Ahlstedt S, Custovic A: Allergy or tolerance in children sensitized to peanut: prevalence and differentiation using component-resolved diagnostics. J Allergy Clin Immunol 2010;125:191-197.
7 Skypala IJ, Bull S, Deegan K, Gruffydd-Jones K, Holmes S, Small I, Emery PW, Durham SR: The prevalence of PFS and prevalence and characteristics of reported food allergy; a survey of UK adults aged 18-75 incorporating a validated PFS diagnostic questionnaire. Clin Exp Allergy 2013;43:928-940.

8 Deinhofer K, Sevcik H, Balic N, Harwanegg C, Hiller R, Rumpold H, Mueller MW, Spitzauer S: Microarrayed allergens for IgE profiling. Methods 2004:32:249-254.

9 Antonicelli L, Massaccesi C, Braschi MC, Cinti B, Bilo MB, Bonifazi F: Component resolved diagnosis in real life: the risk assessment of food allergy using microarray-based immunoassay. Eur Ann Allergy Clin Immunol 2014;46:30-34.

10 Heaps A, Carter S, Selwood C, Moody M, Unsworth J, Deacock S, Sumar N, Bansal A, Hayman G, El-Shanawany T, Williams P, Kaminski E, Jolles S: The utility of the ISAC allergen array in the investigation of idiopathic anaphylaxis. Clin Exp Immunol 2014;177: 483-490.

11 Cabrera-Freitag P, Goikoetxea MJ, Beorlegui C, Gamboa P, Gastaminza G, FernándezBenítez M, Ferrer M, Blanca M, Sanz ML: Can component-based microarray replace fluorescent enzimoimmunoassay in the diagnosis of grass and cypress pollen allergy? Clin Exp Allergy 2011;41:1440-1446.
12 Sampson HA, Ho GH: Relationship between food-specific IgE concentrations and the risk of positive food challenges in children and adolescents. J Allergy Clin Immunol 1997; 100:444-451.

13 Wöhrl S, Vigl K, Zehetmayer S, Hiller R, Jarisch R, Prinz M, Stingl G, Kopp T: The performance of a component-based allergen-microarray in clinical practice. Allergy 2006;61: 633-639.

14 Heinzerling L, Mari A, Bergmann KC, Bresciani M, Burbach G, Darsow U, Durham S, Fokkens W, Gjomarkaj M, Haahtela T, Bom AT, Wöhrl S, Maibach H, Lockey R: The skin prick test - European standards. Clin Transl Allergy 2013;3:3.

15 Focke M, Marth K, Valenta R: Molecular composition and biological activity of commercial birch pollen allergen extracts. Eur J Clin Invest 2009;39:429-436.

16 Martínez-Aranguren R, Lizaso MT, Goikoetxea MJ, García BE, Cabrera-Freitag P, Trellez O, Sanz ML: Is the determination of specific IgE against components using ISAC 112 a reproducible technique? PLoS One 2014;9: e88394.
Allergy Microarray Tests in Patients Presenting Diagnostic Difficulty
Int Arch Allergy Immunol 2017;172:215-223 DOI: $10.1159 / 000464326$ 